\title{
LES PARTIES ET LE TOUT : JAKOBSON, HUSSERL ET LA PHONOLOGIE*
}

\section{Federico Albano Leoni}

\author{
Rome
}

\section{Résumé}

Partant de la lecture de deux écrits de Jakobson, qui se réfèrent explicitement aux Recherches logiques de Husserl et à la question des rapports entre le tout et ses parties, l'auteur critique l'hypothèse d'une racine phénoménologique de la phonologie structurale.

\section{Mots-clés}

Jakobson, Husserl, phonème, phénoménologie

\begin{abstract}
Starting from two articles by Jakobson where Husserl and the relationship between the whole and its parts are explicitly mentioned, this paper criticizes the hypothesis of a phenomenological root of the structural phonology.
\end{abstract}

\section{Keywords}

Jakobson, Husserl, phoneme, phenomenology

\section{PRÉMISSE}

Roman Jakobson est un représentant insigne de la linguistique du XXe siècle, trait d'union entre la linguistique européenne, où il est né, à Moscou et à Prague, et la linguistique américaine. Structuraliste, il se trouve donc à l'intérieur d'un univers complexe, parce que le terme et le concept de «structure » sont au carrefour de plusieurs disciplines du siècle passé 1 .

* Durant l'écriture de cet article j'ai eu l'opportunité d'en discuter avec Giuseppe Di Salvatore, Lia Formigari et Savina Raynaud qui ont été généreux en suggestions précieuses et que je remercie.

1 La bibliographie est immense. Pour le concept de « structure » et de «structuralisme » je me borne ici à citer, outre l'essai fondamental de Piaget (1968), les travaux de Bastide (1962) et Pomian (1981) pour l'ensemble des sciences humaines et sociales ; de Cassirer (1945), Eco (1972 [1968]), Boudon (1968), Holenstein (1974) et Petitot-Cocorda (1985, p. 19-26, 4043) pour les côtés sémiologiques et philosophiques ; de Brøndal (1939), Benveniste (1962), Lepschy (1976 [1966]) et Ducrot (1968) pour le structuralisme linguistique. Lepschy (1962) ébauche la préhistoire du terme structure, de l'organicisme aux néogrammairiens. 
En simplifiant, on peut dire que le concept de "structure " renvoie à l'idée d'un ensemble ordonné, un tout qui n'est pas la somme ou la juxtaposition de ses composantes, de parties pourvues d'existence autonome, mais qui, au contraire, est tel que les parties ne lui préexistent pas et que chacune d'entre elles n'est déterminée que par ses relations avec les autre parties.

Si la question est posée en ces termes, l'on voit que le concept de « structure », comme celui de Gestalt, sont des déclinaisons différentes du problème des rapports entre le tout et les parties et le renvoi aux Recherches logiques de Husserl, et en particulier à la troisième, est donc tout à fait naturel. Comme le suggère le titre que je propose pour cet article, je voudrais présenter quelques réflexions sur la pensée phonologique de Jakobson et sur ses rapports avec la phénoménologie.

Cette association n'est pas infondée non seulement parce que l'on sait (Raynaud 1990, p. 73-75 et passim; De Palo 2010a et b, 2013) que Husserl est présent, directement (par une célèbre conférence à Prague le 1935, dont le texte est malheureusement perdu : $c f$. Raynaud 1990, p. 74), et indirectement, pendant la période des structuralismes européens naissants, à Prague dans les Travaux, avec les articles de Bühler $(1931,1936)$ et de Pos $(1939)^{2}$, et à Copenhague, dans le document programmatique de la revue Acta linguistica (Brøndal 1939), mais surtout parce qu'il existe une ligne interprétative de la pensée de Jakobson, représentée par Holenstein (1974), Petitot-Cocorda (1985) et Coquet (2007), selon laquelle on reconnaitrait dans l'œuvre du linguiste russe une présence si importante de Husserl, que l'on a parlé, bien qu'avec quelque prudence (Holenstein 1974, p. 11), d'un structuralisme phénoménologique.

La question est complexe ${ }^{3}$ et je me promets de l'approfondir ailleurs, en me bornant ici à présenter quelques réflexions sur deux points qui, je le souligne, concernent le Jakobson phonologue stricto sensu. Je crois en fait qu'il y a des éléments qui suggèrent de mettre en discussion le bien-fondé de cette association ${ }^{4}$.

2 Pos (1939) représente, à ma connaissance, la réflexion la plus précoce et approfondie sur le structuralisme menée par un philosophe phénoménologue.

3 Cependant, j'observe que la question de la présence de Husserl et de la phénoménologie dans l'œuvre de Jakobson n'a pas retenu l'attention de deux observateurs très perspicaces : Lepschy (1976 [1966]) l'ignore; Heilmann (1966, p. x, xix-xx) ne lui dédie qu'une mention fugace.

4 En marge il y a à remarquer que Jakobson (1962 [1939], p. 301) ne cite Pos qu'à propos du concept d'opposition (sur lequel je reviendrai) et il ne développe aucun des points saillantes de l'article du philosophe (et en général de la phénoménologie) : il ignore la question de la conscience du sujet parlant et de l'introspection, celle de l'intersubjectivité, qui est garantie par les types idéaux transcendantaux auxquels les parlants se conforment, le finalisme inconscient de la langue. Selon Coquet (2007, p. 19-20) le thème de l'intersubjectivité, discuté par Pos, se manifesterait dans le modèle jakobsonien de la communication. L'affirmation est surprenante parce qu'il me semble que le modèle de Jakobson est décidément plus proche de Shannon que de Husserl. 
Il est certainement vrai que, en ce qui concerne les citations explicites, Husserl est bien présent dans l'œuvre de Jakobson et il suffit de feuilleter les Selected Writings pour s'en apercevoir ${ }^{5}$. Mais il est aussi vrai qu'en général ces citations sont vagues.

Cependant il y a deux cas, qui concernent directement ou indirectement la phonologie, où la citation de Husserl permet quelques observations pertinentes. Le premier est représenté par un long essai sur la structure du phonème (Jakobson 1962 [1939]) ; le second par un bref article sur le rapport entre le tout et les parties en linguistique (Jakobson 1971 [1963]).

\section{LA STRUCTURE DU PHONÈME}

Dans cet essai (1962 [1939]), qui est la summa de la pensée phonologique de Jakobson à la fin de son expérience pragoise, où les prémisses du binarisme apparaissent déjà avec clarté, le savant pose avec courage une question généralement contournée par les phonologies : s'il est vrai que dans la perspective saussurienne (envers laquelle Jakobson est souvent critique, mais dont il semble accepter les fondements sémiologiques) les unités linguistiques sont les signes, c'est-à-dire des entités qui consistent dans l'union indissoluble d'un signifiant et d'un signifié, comment se peut-il que le phonème soit une unité linguistique, étant donné qu'il est dépourvu de signifié ?

En fait le Cours est très clair à ce sujet :

L'entité linguistique n'existe que par l'association du signifiant et du signifié ; dès qu'on ne retient qu'un de ces éléments, elle s'évanouit ; au lieu d'un objet concret, on n'a plus devant soi qu'une pure abstraction. À tout moment on risque de ne saisir qu'une partie de l'entité en croyant l'embrasser dans sa totalité ; c'est ce qui arriverait par exemple, si l'on divisait la chaine parlée en syllabes ; la syllabe n'a de valeur qu'en phonologie. Une suite de sons n'est linguistique que si elle est le support d'une idée ; prise en elle-même elle n'est plus que la matière d'une étude physiologique (Saussure 1962 [1916], p. 144).

Pour répondre à la question que je viens de poser en suivant Saussure, il n'y a que deux voies : ou bien l'on renonce décidemment à considérer le phonème comme une unité linguistique - c'est ma position (Albano Leoni 2007), et contrairement à l'opinion de Jakobson, c'est aussi ce que pensait Saussure - ; ou bien il faut

5 «Si l'on suit les citations explicites, on constate une influence directe de Husserl [...] » (Holenstein 1974, p. 9). Holenstein a raison, mais je ne sais pas quel poids on pourrait assigner au fait que dès que Jakobson s'installe aux États-Unis la présence de Husserl s'affaiblit jusqu'à la disparition, substituée par une présence croissante de Pierce. Lia Formigari me signale que selon Koerner (« Remarks on the Sources of R. Jakobson's Linguistic Inspiration », Cahiers de l'ILSL, 9, 1997, p. 151-168) les citations de Husserl témoigneraient d'une découverte a posteriori de quelques affinités plutôt que des apports constitutifs pour la construction de sa théorie. 
trouver un escamotage. La première de ces voies étant évidemment impossible pour un des pères de la phonologie, il ne restait que la seconde. L'argumentation de Jakobson est articulée en deux points.

Dans le premier point Jakobson, qui d'ailleurs prend souvent ses distances avec le maître genevois et son école, reconnait à Saussure (et à Baudouin de Courtenay) le mérite d'avoir soustrait la phonétique au chaos de la variation infinie de la matière phonique, en lui reconnaissant sa fonction signifiante et en ramenant donc la Lautform à sa fonction primordiale, celle de la signification :

Zwei geniale Sprachforscher, Baudouin de Courtenay und Ferdinand de Saussure, rollten die Frage nach dem Zwecke der Sprachklänge auf, und das Studium des lautlichen Feldes der Sprache unter dem Gesichtspunkt der sprachlichen Funktionen wurde von ihren Schülern und Nachfolgern eingeleitet. Die Lautform der Sprache, die bis dahin ein blosser Gegenstand der Sinnespsychologie und -physiologie war, wurde endlich der Linguistik im wahren Sinne des Wortes einverleibt, d.h. die Lautform wurde unter dem Gesichtspunkt ihres Zeichenwertes und vor allem ihrer bedeutungsverleihenden Funktion [italiques F. A. L.] untersucht. Die massgebliche Frage, das "wozu" der Sprachlaute, d.h. ihre unmittelbare raison d'être kam endlich zur Geltung (Jakobson 1962 [1939], p. 280-281).

Deux linguistes géniaux, Baudouin de Courtenay et Ferdinand de Saussure, posèrent la question du but des sons du langage et leurs élèves et successeurs introduisirent l'étude du champ phonique de la langue du point de vue des fonctions linguistiques. La forme phonique de la langue, qui jusqu'à ce momentlà n'avait été que l'objet de la psychologie et de la physiologie sensorielles, fut finalement incorporée dans la linguistique dans le vrai sens du mot, c'est-à-dire que la forme phonique fut investiguée du point de vue de sa valeur sémiologique et surtout du point de vue de sa fonction conférant un sens [italiques F. A. L.]. La question de la finalité des sons du langage, de leur raison d'être immédiate se fit enfin valoir. [Traduction F. A. L.]

Jakobson se réfère ici à la Lautform (qu'il appellera plus tard sound shape), qui est, pour en rester à Saussure, le signifiant phonique en général. En outre, il lui attribue une bedeutungsverleihende (Funktion), c'est-à-dire une «(fonction) qui confère une signification », et la référence à la terminologie husserlienne, sur laquelle je reviendrai tout de suite, est bien évidente.

Par ce choix terminologique Jakobson introduit le deuxième point de son argument, qui concerne non plus la Lautform en général, mais le phonème dans son sens désormais technique, qui est, comme Jakobson le sait très bien, autre chose que la Lautform. Ainsi, après avoir réglé la discussion sur l'ontologie du phonème (p. 281-282), qu'il juge oiseuse, Jakobson propose de résoudre la difficulté théorique de conférer au phonème, quoique dépourvu de sens en soi, le statut d'unité linguistique, en s'appropriant explicitement une distinction husserlienne et en appliquant au phonème ce qu'il venait de dire de la Lautform : 
Auch ein Phonem ist in diesem Sinne doppelseitig, doch das Eigenartige und Seltsame liegt hier darin, dass dem bestimmten und konstanten lautlichen Unterschied zweier Phoneme die blosse Tatsache eines potentiellen Bedeutungsunterschiedes, keinesfalls aber ein bestimmter und konstanter Bedeutungsunterschied entspricht. Um mit Husserl zu sprechen, ist im Phonem der bedeutungsverleihende Akt, keineswegs aber der bedeutungserfüllende Akt gegeben. (Jakobson 1962 [1939], p. 292).

Le phonème aussi est dans ce sens à double face, mais ce qu'il y a de particulier et de singulier est que à la différence phonique certaine et constante entre deux phonèmes ne correspond que le simple fait d'une distinction sémantique potentielle et nullement une distinction sémantique également certaine et constante. Pour le dire avec Husserl, dans le phonème est donné l'acte qui confère une signification, mais nullement l'acte qui remplit la signification. [Traduction F. A. L.]

Il est tout à fait évident que la référence est à Husserl (1961 [1913], vol. 2, I, p. 42-44) qui en effet distingue entre « actes conférant la signification » et « actes remplissant la signification ». Il sera utile de lire directement quelques passages tirés des pages citées.

Husserl écrit (ibid., p. 42-43) :

Si nous nous plaçons sur le terrain de la description pure, le phénomène concret de l'expression animée d'un sens (sinnbelebten) s'articule comme suit : d'une part, le phénomène physique où l'expression se constitue selon son aspect physique, et, d'autre part, les actes qui lui donnent la signification, et, éventuellement, sa plénitude intuitive, et où se constitue sa référence à une objectité exprimée.

Et peu avant (ibid., p. 43-44), il précise :

Si nous prenons pour base cette distinction fondamentale entre intentions de signification vides d'intuition et celles qui sont remplies, il faut aussi distinguer, après avoir mis de côté les actes sensibles dans lesquels se réalise l'apparition de l'expression en tant que complexe phonique, entre deux espèces d'actes ou de séries d'actes : d'une part, ceux qui sont essentiels à l'expression, pour autant qu'elle doit rester encore expression, c'est-à-dire complexe phonique animé d'un sens ; ce sont ces actes que nous appelons les actes conférant la signification, ou intentions de signification. D'autre part, les actes qui ne sont sans doute pas essentiels à l'expression comme telle, mais qui se trouvent néanmoins avec elles dans ce rapport logique fondamental, qu'ils remplissent (confirment, renforcent, illustrent) son intention de signification d'une manière plus ou moins adéquate, et qu'ainsi ils actualisent précisément sa référence objective. Ces actes qui, dans l'unité de connaissance ou de remplissement, fusionnent avec les actes conférant la signification, nous les appelons actes remplissant la signification.

L'extension de ces catégories husserliennes à la catégorie structurale du phonème est-elle légitime? Je ne le crois pas.

Avant tout, il faut noter que là où Husserl parle des actes conférant la signification, il ne considère jamais des unités minimales (concept qui d'ailleurs lui est étranger) asémantiques parce que les actes dont il parle ont pour objet «le 
phénomène concret de l'expression animée d'un sens (sinnbelebten) » (ayant explicitement mis de côté « les actes sensibles dans lesquels se réalise l'apparition de l'expression en tant que complexe phonique », qui ne sont que la manifestation de la matière phonique non formée ni découpée). En fait Husserl envisage ici, je le répète, les actes « qui sont essentiels à l'expression, pour autant qu'elle doit rester encore expression, c'est-à-dire complexe phonique animé d'un sens ». Or, quelle que soit la définition qu'on veut donner du phonème, il me semble difficile de lui attribuer la propriété d'être animé par un sens et encore plus difficile de le considérer comme un complexe (à moins de vouloir interpréter " complexe » comme un faisceau de traits distinctifs, ce qui serait encore pire, comme on le verra après).

Somme toute, tandis qu'il semblerait acceptable d'établir une analogie entre la Lautform de Jakobson et le Wortlaut ou, mieux, le Wort de Husserl, il ne semble pas qu'il y en ait une entre le phonème de Jakobson et des structuralistes, d'un côté, et l'appareil conceptuel de Husserl, de l'autre 6 .

Enfin, la lecture de la première recherche sur expression et signification (ibid., p. 25-121), et en particulier du chapitre II (Les caractéristiques des actes conférant la signification, p. 70-88), montre aisément qu'il est impossible que le phonème se range parmi une des catégories qui y sont analysées et donc qu'il puisse être considéré comme un acte. Il suffit de lire les lignes qui ouvrent ce chapitre pour s'en convaincre (p. 70) :

Nous avons situé le concept de signification, ou encore celui d'intention de signification, d'après le caractère phénoménologique qui est essentiel à l'expression comme telle, et qui la distingue dans la conscience, par conséquent descriptivement, du simple complexe phonique. D'après notre théorie, ce caractère peut exister et existe assez souvent sans que l'expression ait une fonction de connaissance ni un rapport, si lâche et si éloigné soit-il, avec les intuitions qui rendent sensible la signification.

L'idée donc que le phonème (qui n'est pas du tout le complexe phonique dont parle Husserl) entre dans cet univers de discours et puisse conférer une signification semble être tout à fait étrangère à la pensée de Husserl.

Par conséquence, l'aporie générée par le choix de considérer les phonèmes asémantiques comme des unités linguistiques reste irrésolue même dans une perspective phénoménologique.

De ce que je viens de dire, je tirerai la conclusion que cette référence à Husserl est si superficielle qu'elle n'est pas recevable. Et en effet, dans cet article, Jakobson abandonne rapidement l'argument husserlien pour passer au concept bühlerien de Zeichen am Zeichen, que je ne développerai pas ici parce que cela nous amènerait vers un autre problème, à savoir celui des rapports entre Jakobson et Bühler.

6 Giuseppe Di Salvatore (communication privée) me fait observer que Jakobson ne sait pas (ou ne peut pas) coordonner une bedeutungsvolle Lautform avec un bedeutungsloses Phonem. 
Le fait est que Jakobson ne se mesure qu'en surface avec le grand problème du rapport entre le tout et les parties, comme on le verra dans la prochaine section.

\section{LE TOUT ET LES PARTIES}

Le deuxième texte de Jakobson, où la référence a Husserl est ponctuelle, est un article de 1963, dont le titre est explicitement husserlien (Parts and Wholes in Language). Ici il semble que Jakobson veuille appliquer des principes husserliens et méréologiques à la description des caractères généraux des langues à partir de la troisième recherche logique (Husserl 2005 [1922]), dédiée justement aux entiers et aux parties, et d'un important essai de Nagel (1963 [1952] ; 1979).

L'article de Jakobson s'ouvre par une déclaration de foi phénoménologique, qui est en même temps un reproche à la linguistique de n'avoir pas prêté une attention suffisante au problème général du rapport entre le tout et les parties.

In the second part of Edmund Husserl's Logische Untersuchungen, still one of the most inspiring contributions to the phenomenology of language, two studies devoted to "Wholes and Parts" introduce the philosopher's meditations on "the Idea of Pure Grammar". In spite of the manifold aspects of interdependence between wholes and parts in language, linguists have been prone to disregard this mutual relationship (Jakobson 1971 [1963], p. 280).

Je ne suis pas sûr que le reproche soit tout à fait justifié, mais en principe on ne peut que partager l'exhortation à considérer avec attention les rapports entre les éléments (les parties) qui constituent la langue (le tout), ou entre les énoncés ou les textes (le tout) et les éléments (les parties) dont ils sont composés. Il faut pourtant se demander si Jakobson lui-même a vraiment introduit cette réflexion dans son travail et encore s'il l'a développée avec cohérence ${ }^{7}$.

Cependant, puisqu'il n'est pas difficile à comprendre que la distance entre les Recherches logiques de Husserl (et son idée d'une grammaire pure) et n'importe

7 Dans ce même article Jakobson reproche à la linguistique d'autres graves omissions et en fait il se plaint du fait qu'il y a une "nearly unexplored question of the interrelation between message and context » (p. 282), et que " the structural laws of ellipsis have not yet been subjected to a thorough analysis » (ibid.). Les reproches sont surprenants. Il est très difficile de croire que Jakobson ignorait les œuvres de Wegener (1991 [1885]), Brugmann (1904), Malinowski (1923), Gardiner (1989 [1932]), Bühler (2009 [1934]), qui toutes ont pour cible le fait que le contexte, la situation sont partie intégrante de l'activité langagière (et je n'hésite pas à juger stupéfiante l'omission de Bühler, auquel Jakobson doit beaucoup plus qu'il ne reconnait). Il en va de même pour la question de l'ellipse, où Jakobson oublie, ou ignore, non seulement, et encore, Bühler (qui a écrit sur l'ellipse des pages très aiguës), mais aussi les Recherches philosophiques de Wittgenstein (2005 [1953]). Ailleurs (Albano Leoni 2011) j'ai souligné que l'attitude de Jakobson envers Bühler n'est pas limpide. Sur le thème de l'ellipse cf. Mulligan (2004) et De Palo (2010a, 2013). Enfin, Mathesius (1911), peu lu en Occident (et récemment reproposé à l'attention des savants par Raynaud 2013 : cf. Mathesius 1911), aurait dû être connu par Jakobson. 
quelle description structurale ou autre d'un aspect ou d'un mécanisme des langues est très grande, Jakobson abandonne vite Husserl et, dans la suite de l'article, suit la trace qui lui vient d'un essai d'un grand épistémologue (Nagel 1963 [1952]), en adaptant les espèces de relations tout/parties décrites par Nagel (dans la dimension spatiale, ou temporelle, ou dans la relation entre ensembles et objets qu'en font partie) à des exemples linguistiques.

Ainsi, dans la relation entre un processus (le tout) et des parties d'un processus (qui sont à leur tour des processus), Jakobson voit la même relation qu'entre un événement linguistique (le tout) et ses composantes psychophysiques (les parties).

Dans la relation entre un tout comme extension temporelle et les parties qui en sont les portions, elles aussi pourvues d'extension temporelle, il voit la même relation que l'on trouve dans l'analyse d'un énoncé (le tout) en constituants immédiats (les parties) du distributionnalisme américain.

Dans la relation entre classes (le tout) et objets qui en font partie Jakobson voit une analogie entre les catégories linguistiques (le tout) et les objets qu'on leur assigne ${ }^{8}$, et enfin dans la relation entre un objet (le tout) et ses propriétés (les parties) il voit la relation entre un phonème et ses traits.

From the sentence model as a whole we pass to various syntactic patterns of sentences, on the one hand, and to the grammatical constituents of the sentence, on the other. When we reach the level of the word, then either word classes or, again the morphological constituents of the word serve as parts. Gradually we arrive at the ultimate stage - the analysis of the smallest meaningful units into distinctive features. An important structural particularity of language is that at no stage of resolving higher units into their component parts does one encounter informationally pointless fragments (Jakobson 1971 [1963], p. 283).

Je passe sur l'affirmation surprenante que les unités minimales, c'est-à-dire les phonèmes, seraient meaningful, parce qu'il s'agit peut-être d'un lapsus calami. Par contre j'observe qu'en établissant ces analogies Jakobson fait des simplifications ou, mieux, il omet de mettre en lumières le noyau problématique et les difficultés très sérieuses, au moins du point de vue de la logique et de la méréologie, qui, une fois convoquées, exigent que leur règles soient respectées.

À titre d'exemple je me servirai d'un article de Hammond (2001-2002), où il examine quelques problèmes posés par les travaux de trois grands épistémologues, à savoir Nagel (1963 [1952], 1979), Polanyi (1958) et Simons (1987).

Hammond mène une simulation, à la fois amusante et provocante, de l'analyse d'un objet concret. Soit une boîte de soupe (un tout) : elle peut être partagée en portions (les parties) ; chaque portion (un tout) peut être partagée en cuillérées (les parties) ; encore la soupe (le tout) peut être analysée en ses composants (les parties), dont par exemple une pomme de terre (une partie) ; la pomme (le tout) à son tour peut être analysée en ses composants chimiques (les parties), dont les

8 Cette troisième relation est in absentia, paradigmatique, donc asymétrique par rapport aux autres qui sont in praesentia, syntagmatiques. 
uns sont les carbohydrates. Arrivés à ce point-ci, on est en face d'une question indécidable, à savoir si les carbohydrates sont une partie de la pomme ou si la pomme concourt, comme partie, à former l'ensemble des carbohydrates (comme classe, c'est-à-dire comme un tout).

La filière qui mène de la boîte de soupe aux carbohydrates est tout à fait analogue à celle représentée par Jakobson dans le passage que je viens de citer, mais, en proposant l'analogie, il s'arrête ante portas, parce que ce qu'il propose n'est qu'un exercice de découpage mécanique, qui ne tient aucun compte du statut ontologique et phénoménologique des éléments qui représentent alternativement le tout ou la partie. Parce qu'en fait il est vrai qu'en appliquant cette procédure à l'analyse d'un énoncé on peut arriver jusqu'aux traits, mais qu'est-ce qu'il se passe quand on arrive au bout?

Je propose l'exemple de l'opposition privative (Troubetzkoy 1949 [1939], p. 77) dans laquelle « un des termes de l'opposition est caractérisé par l'existence d'une marque, l'autre par l'absence de cette marque » : le premier est dit « marqué » et le second est dit « non marqué ». Donc la paire de phonèmes français $/ \mathrm{p} / \mathrm{vs} / \mathrm{b}$ / (p. ex. dans la paire minimale poule/boule) est caractérisée par le fait que, dans le schéma binaire de Jakobson, le terme marqué /b/ est [+voisé] (ce qui correspond à la présence d'une vibration glottale et à une oscillation quasi-périodique des molécules d'air), et le terme non marqué /p/ [-voisé], est dépourvu de cette vibration ${ }^{9}$.

Or, revenant à l'interprétation (husserlienne ?) du rapport entre le tout et les parties, tel que le propose Jakobson dans le cas de l'opposition privative, je crois qu'il faut se demander quel est le statut du trait [-voisé], c'est-à-dire d'un trait qui n'a aucun corrélat physique positif et qui ne puise sa légitimation que dans une construction logique du niveau méta-métalinguistique. En fait, il n'est pas aisé de comprendre comment une partie qui n'existe pas (le trait [-voisé] dans la représentation de Jakobson) peut être la partie d'un tout : une opposition privative n'a de consistance ni sur le plan ontologique, ni sur le plan cognitif. Enfin on ne peut accepter non plus que certaines des parties d'un tout (c'est-à-dire certains des traits qui devraient concourir à former un phonème) manifestent des propriétés positives et d'autres des propriétés corrélatives.

9 J'ajouterai, sans entrer maintenant dans les détails de la question, que je crois que nous reconnaissons un son d'après ses propriétés positives, et je refuse donc l'interprétation mécanique du principe saussurien que dans la langue il n'y a que des différences (Saussure 1962 [1916], p. 68-69, relativement aux sons), qui nous amènerait à conclure que je ne reconnais un objet qu'après avoir établi qu'il n'est pas son contraire. En fait, Saussure lui-même dit que le principe de la différence, au moins en ce qui concerne les sons, n'est qu'un principe de classification (p. 71), dépourvu de tout intérêt théorique (1967-68, fasc. 1, p. 817-818 : D 67, SM III 104, S. 1.36 ; j. 59). Or, une chose est la classification des sons, activité métalinguistique explicite, autre chose est l'activité langagière. 
On pourra rétorquer que Husserl, dans la troisième recherche, prend en compte aussi des

généralités « analytiques » pures comme : il ne peut y avoir de roi, de maître, de père, s'il n'y a pas de sujets, de serviteurs, d'enfants, etc. Ce qui veut dire ici, d'une manière générale : des termes corrélatifs se postulent l'un l'autre réciproquement, ils ne peuvent être pensés ni exister l'un sans l'autre (1961, 2, II, p. 36),

mais cette forme de présupposition réciproque ne peut aucunement être identifiée avec la logique de l'opposition privative ou de l'opposition binaire en général, ne fût-ce que par le fait qu'un imaginaire "non père" n'est pas égal à "fils" (tandis que pour Jakobson au moins en principe le trait [-voisé] ne peut se relier qu'au trait [+voisé] et, en général, n'importe quel trait ne peut se relier qu'à son contraire).

On peut observer, entre parenthèses, que même Jakobson (1962 [1939], p. 301), tout en acceptant une proposition de Pos sur les oppositions

« [...] ein wirkliches Oppositionsglied kann nicht ohne das andere Glied gedacht werden. L'un implique l'autre, nach dem treffenden Satz des hervorragenden holländischen Sprachphilosophen H. J. Pos »

le membre d'une véritable opposition ne peut être pensé sans l'autre membre. L'un implique l'autre, comme le dit d'une manière efficace l'éminent philosophe hollandais H. J. Pos [Traduction F. A. L.]

était alors très prudent quant aux conséquences logiques du principe oppositif et, en se référant explicitement aux exemples de Pos sur les couples polaires (antinomiques) du type beau/laid etc. ${ }^{10}$, il exprime ses doutes à propos des oppositions phonologiques telles que /a/ vs /u/, qui sont complexes :

Die mannigfaltigen Oppositionsbegriffe sind in einer Hinsicht gleich: die Begriffe Vater und Mutter, Tag und Nacht, teuer und billig, gross und klein setzen einander voraus. Bei den Phonemen $/ \mathrm{u} / \mathrm{und} / \mathrm{a} /$ ist das nicht der Fall. Soll das bedeuten, dass man das Phonemverhältnis nur ungenau als Opposition bezeichnet, und das man hier mit blossen Differenzen, dualités contingentes, und keineswegs mit echten Oppositionen zu tun hätte? Ich lasse einstweilen diese Frage offen (p. 301).

Les nombreux concepts d'opposition sont tous pareils, d'un certain point de vue : les concepts de père et mère, jour et nuit, cher et bon marché, grand et petit, se présupposent réciproquement. Mais il en va autrement pour les phonèmes $/ \mathrm{u} /$ et $/ \mathrm{a} /$. Est-ce que cela veut dire que la dénomination d'opposition pour les rapports entre les phonèmes est inexacte, et qu'ici on a affaire à de simples différences, des dualités contingentes, plutôt qu'à de véritables oppositions ? Pour le moment je laisse ouverte cette question. [Traduction F. A. L.]

10 J'observe que telles oppositions n'ont cours que dans les usages du langage ordinaire et dans le sens commun, parce que, comme nous allons voir, du point de vue de la logique binaire l'opposé de « beau » n'est pas « laid» mais est « non-beau ». 
Les réflexions que je suis en train de proposer sur le statut du binarisme et des traits négatifs me poussent à manifester mes doutes à propos de ce que PetitotCocorda (1985, p. 38-39) écrit en essayant d'interpréter, à mon avis d'une façon impropre, les traits binaires de Jakobson comme la manifestation phonologique du principe husserlien de fondation (Fundierung) :

Ce dernier problème, à savoir celui des rapports de dépendance (aussi dits rapports de fondation) entre un moment et le tout dont il est le moment non détachable, a fait l'objet d'investigations approfondies tant de la part de Stumpf et de Meinong que de Husserl [...] On ne saurait en sousévaluer l'importance puisque [...] il se trouve à l'origine de la phonologie jakobsonienne, les traits distinctifs étant par excellence des moment dépendants : les phonèmes ne sont ni des classes d'équivalence d'allophones, ni des artefacts descriptifs, ni des abstracts, mais des unités formelles et relationnelles constituées par des rapports de fondation, rapports réels au sens d'une autonomie ontologique du niveau phonologique ${ }^{11}$.

Ceci est, me semble-t-il, le passage clé pour la construction d'une phonologie phénoménologique parce qu'y est formulée l'hypothèse que les traits binaires d'un phonème stipulent entre eux un rapport de fondation. Mais ce passage et cette hypothèse requièrent un commentaire.

Avant tout, je crois qu'il sera utile nous rappeler ce que Husserl envisage quand il définit la fondation (1961 [1913], vol. 2, II, p. 45-46) :

$\mathrm{Si}$, conformément à une loi d'essence, un $\alpha$ ne peut exister comme tel que dans une unité qui l'embrasse et qui le relie avec un $\mu$, nous disons qu'un a comme tel a besoin d'être fondé par un $\mu$, ou encore qu'un $\alpha$ comme tel a besoin d'être complété par un $\mu$. Si, en conséquence, $\alpha^{\circ}, \mu^{\circ}$ sont des cas singuliers déterminés, réalisés dans un seul tout, des genres purs $\alpha$ ou $\mu$, se trouvant dans le rapport indiqué, nous dirons que $\alpha^{\circ}$ est fondé par $\mu^{\circ}$ et exclusivement fondé par $\mu^{\circ}$ quand seul $\mu^{\circ}$ peut satisfaire ce besoin d' $\alpha$ d'être complété.

On pourrait dire qu'on est ici en face d'une relation de présupposition et on a observé que dans le domaine des catégories linguistiques cette relation n'est observable à la rigueur qu'entre les deux faces du signe, et certainement elle ne l'est pas dans les relations entre les traits de n'importe quelle matrice binaire et on ne pourra jamais affirmer que, par exemple, le besoin d'intégration d'un trait donné est satisfait uniquement par un et un seul autre trait. Donc il me semble que la fondation envisagée par Petitot et la fondation définie par Husserl ne sont pas la même chose.

11 Je passe ici sur le concept d' " autonomie ontologique du niveau phonologique », qui est une aporie si, comme je le présume, l'auteur connaît la valeur des termes de la phonologie classique. Sans doute la matière phonique jouit d'une existence autonome. Mais dès le moment où cette matière est formée, entrant dans la dimension phonologique, elle perd toute autonomie parce qu'en tant que forme linguistique elle n'existe que dans le lien avec un sens. 
Je voudrais ajouter que je crois fermement que le point de départ pour une interprétation de la pensée et du modèle phonologiques de Jakobson est le texte où ce modèle est présenté dans sa forme mûre et définitive, donc dans les Foundamentals of language (1956, avec Halle) qui est le texte où la théorie générale est décrite analytiquement.

Or, de ce texte on apprend en premier lieu que le modèle général adopté et prisé par Jakobson est celui de Bloomfield selon lequel les traits sont des propriétés physiques et les phonèmes sont des ensembles de traits, dans le sens d'un agrégat ou d'un faisceau (bundle) et non d'une structure. On lit (Jakobson et Halle 1956, p. 8) :

This so-to-speak inner, immanent approach [scil. celui de Bloomfield], which locates the distinctive features and their bundles within the speech sounds, be it on their motor, acoustical or auditory level, is the most appropriate premise for phonemic operations, although it has been repeatedly contested by outer approaches which in different ways divorce phonemes from concrete sounds ${ }^{12}$.

Il me semble ici que la terminologie et la conceptualisation de Jakobson sont très éloignées de la perspective husserlienne.

Deuxièmement il faut observer que la nature binaire des traits, selon laquelle, je le répète, chaque trait est en opposition avec son contraire, est affirmée avec fermeté (sans les doutes que Jakobson avait manifestés en 1939), et que cette opposition est inhérente au système (nommé code) (p. 26) :

[...] both alternatives of an inherent feature co-exist in the code as two terms of an opposition, but do not require a contrasting juxtaposition within one message. Since the inherent feature is identified only through the comparison of the alternative present in the given position with the absent alternative, the implementation of an inherent feature in a given position admits less variability than that of the prosodic features.

Or, cette géométrie n'est régulière qu'en apparence parce que les traits et leurs oppositions binaires n'ont pas tous le même statut : à coté des paires proprement binaires, comme dans le cas d'une opposition privative, on trouve des paires où les traits s'opposent par un degré différent d'une propriété mesurable.

C'est le cas, par exemple, des oppositions [+vocalique/-vocalique] et [+consonantique/-consonantique] qui peuvent se trouver en contraste avec le principe binaire parce que le système accepte des phonèmes caractérisés comme [+vocalique, +consonantique] et [-vocalique, -consonantique] :

Vowels are vocalic and non-consonantal; consonants are consonantal and non-vocalic; liquids are vocalic and consonantal (with both free passage and obstruction in the oral cavity and the corresponding acoustical effect); glides are non-vocalic and non-consonantal (p. 29).

12 Qu'il s'agit de faisceaux est affirmé encore p. 20: "The distinctive features are aligned into simultaneous bundles called phonemes ». 
Un autre exemple en est l'opposition grave/aigu qui n'est pas en mesure de représenter les voyelles moyennes et doit donc être doublée en [+grave/-grave] et [+aigu/-aigu], ce qui provoque une asymétrie parce que lorsqu'il ne peut y avoir aucun phonème qui soit [+grave, +aigu], il y en a qui sont [-grave, -aigu]. On voit donc que cette logique binaire rudimentaire, empruntée vraisemblablement à la naissante science des ordinateurs (circuit ouvert/fermé) n'est pas en mesure de représenter les complexes manifestations phoniques des langues.

Enfin, il faut se demander encore si tout cela est compatible avec le concept husserlien de fondation. Autrement dit, peut-on affirmer des relations entre les traits, telles que Jakobson les présente, ce que Husserl (1961, vol. 2, II, p. 12-15), en citant Stumpf, dit du rapport de fondation exemplifié dans la relation entre objet, couleur et surface $?^{13}$ Je ne le crois pas, pour deux raisons.

La première résulte de l'observation que le statut (onto)logique des traits est différent du statut des catégories de "couleur" et "surface". Ce qui pourrait valoir, par exemple, pour la relation entre amplitude et fréquence d'une oscillation (sonore ou d'autre nature) parce que l'amplitude ne peut être connue et ne peut exister que dans une fréquence et vice-versa, ne vaut pas pour les traits.

En fait la couleur et la surface, par exemple d'un tissu, sont des catégories génériques (la couleur peut être rouge ou verte, la surface grande ou petite, ronde ou carrée, tout comme sont génériques les catégories de fréquence et de amplitude. Par contre, les traits sont des catégories spécifiques qui réfèrent à des propriétés qui se définissent par le mesurage (p. ex., dans l'opposition aigu/grave le référent n'est pas une fréquence générique mais est une fréquence qui doit être obligatoirement mesurée et confrontée avec une autre).

La deuxième raison est qu'établir une équivalence entre la relation qui relie les traits et le rapport de fondation se heurte à une proposition très claire de Husserl qui, dans le $\S 48$ de la sixième recherche logique (Les actes catégoriaux caractérisés comme actes fondés) affirme à ce sujet :

La perception vise justement à appréhender l'objet lui-même, et ainsi son "appréhension" doit atteindre dans et avec l'objet tout entier toutes ses parties composantes.

Naturellement, il ne s'agit ici que des parties composantes de l'objet comme il apparaît dans la perception et tel qu'il se trouve en elle-même, et non de celles qui appartiennent à l'objet existant dans la "réalité" objective, et que seules des expériences ultérieures, des connaissances, des sciences, nous permettent de dégager (Husserl 1963 [1921], p. 187).

En fait, aucun des traits ne peut être saisi comme élément constitutif de l'objet phonème, parce que leurs propriétés articulatoires ou acoustiques ne sont pas perceptibles analytiquement et ne peuvent être connues que par une analyse instrumentale et donc n'appartiennent pas à la catégorie des éléments constitutifs

13 Cet exemple de Husserl, bien connu, est cité par Holenstein (1974, p. 91) pour démontrer l'équivalence de la relation de fondation avec la relation qui relie les traits. 
de l'objet entre lesquels subsiste un rapport de fondation tel que nous l'observons entre couleur et surface dans la perception d'un pan de tapisserie. Affirmer qu'entre les traits subsiste un rapport de fondation équivaut à affirmer qu'un tel rapport existe entre les atomes d'hydrogène et les atomes d'oxygène quand ils s'unissent pour former la molécule d'eau. Que l'eau soit un composé est un problème chimique mais certainement non phénoménologique ou épistémologique. La situation du phonème n'est pas différente.

\section{CONCLUSIONS}

La première conclusion que je tirerai est que le statut du phonème reste incertain même par rapport à la tentative de lui fournir un soutien phénoménologique. Reste en effet sans solution la question incontournable de la nature asémantique de cette unité et la tentative de voir dans le phonème un acte qui confère un signifié semble faiblement fondé.

La deuxième conclusion est que le statut logique du binarisme et de la théorie des traits, si bien reçu et populaire qu'il soit, apparait, dès qu'on l'observe de près, riche en contradictions et obscurité et surtout, en ce qui nous concerne ici, inadéquat à représenter le concept phénoménologique de fondation.

La troisième conclusion, qui porte sur le thème principal de cet article, résumé dans le titre, est que l'adhésion de Jakobson à la phénoménologie semble plutôt de surface que de substance, visant à la recherche ou à l'exhibition d'une légitimation théorique plutôt qu'à la compréhension profonde de ses présupposés dans le domaine d'une théorie de la connaissance. Ce fait apparait avec évidence soit par les tentatives menées par les exégètes de Jakobson de reconnaitre dans le concept husserlienne de fondation la racine de la phonologie binariste, soit par la tentative menée par Jakobson lui-même de traduire dans une pratique descriptive les principes méréologiques qu'il tire des écrits de Nagel (1963 [1952]).

La cause de l'échec de cette opération est, à mon avis, à chercher dans le fait, particulièrement évident à propos de la phonologie, que les recherches logiques de Husserl portent sur la question centrale de réussir à rendre compte du lien fondateur entre la matière de l'expression, l'expression et ce qui est exprimé. Elles sont donc une théorie de la connaissance et du sujet connaissant et non les préliminaires d'une pratique analytique linguistique ou autre. 


\section{BIBLIOGRAPHIE}

Albano Leoni, Federico, 2007. «Saussure, la syllabe et le phonème», Histoire Épistémologie Langage XXIX-1, 115-136.

- 2011. «Attualità di Bühler », Paradigmi. Rivista di critica filosofica XXIX (3), 125-139.

Bastide, Roger (éd.), 1962. Sens et usages du terme « structure » dans les sciences humaines et sociales, La Haye, Mouton.

Benveniste, Émile, 1962. " "Structure" en linguistique», in Bastide 1962 (repris dans Benveniste, Émile, 1966, Problèmes de linguistique générale 1, Paris, Gallimard, 9198).

Boudon, Raymond, 1968. À quoi sert la notion de "structure »?, Paris, Gallimard.

Brøndal, Viggo, 1939. « Linguistique structurale », Acta Linguistica. Revue internationale de linguistique structurale I, 2-10.

Brugmann, Karl, 1904. Die Demonstrativpronomina der indogermanischen Sprachen. Eine bedeutungsgeschichtliche Untersuchung, Leipzig, Teubner.

Bühler, Karl, 1931. "Phonetik und Phonologie », TCLP 4, 22-53.

- 2009 [1934]. Sprachtheorie. Die Darstellungsfunktion der Sprache, Jena, Fischer (trad. franç. Théorie du langage. La fonction représentative du langage, Marseille, Agone).

- 1936. « Das Strukturmodell der Sprache », TCLP 6, 3-12.

Cassirer, Ernst, 1945. « Structuralism in Modern Linguistics », Word I, 99-120.

Coquet, Jean-Claude, 2007. Phusis et Logos. Une phénoménologie du langage, Paris, PUV.

De Palo, Marina, 2010a. « Le "je”, la phénoménologie et le discours : Bühler, Benveniste et Husserl », Beiträge zur Geschichte der Sprachwissenschaft 20, 155-165.

- 2010b. "Sujet cognitif et sujet linguistique », Histoire Epistémologie Langage, XXXII-2, 37-55.

- 2012. "Vaghezza, strutturalismo e fenomenologia del linguaggio », Thornton, Anna Maria et Voghera, Miriam (éd.), Per Tullio De Mauro, Roma, Aracne, 59-79.

- 2013. "L'ellipse en contexte », Paradigmi. Rivista di critica filosofica XXXI (1), 169180.

Ducrot, Oswald, 1968. Qu'est-ce que le structuralisme ? 1. Le structuralisme en linguistique, Paris, Seuil.

Eco, Umberto, 1972 [1968]. La structure absente. Introduction à la recherche sémiotique, Paris, Mercure de France.

Gardiner, Alan H., 1989 [1932]. Langage et acte de langage : aux sources de la pragmatique, trad. et préf. par Catherine Douay, Villeneuve d'Ascq, Presses Universitaires de Lille.

Hammond, Percy, 2001-2002. " Parts and Wholes. Contrasting Epistemologies », The Polanyi Society Periodical XXVIII (3), 20-27.

Heilmann, Luigi, 1966. "Introduzione », in Jakobson, Roman, Saggi di linguistica generale, Milano, Feltrinelli, vii-xxv.

Holenstein, Elmar, 1974. Jakobson ou le structuralisme phénoménologique, Paris, Seghers.

Husserl, Edmund, 1961 [1913]. Recherches logiques (I-V), Paris, PUF.

- 1963 [1921]. Recherches logiques. Tome troisième : Éléments d'une élucidation phénoménologique de la connaissance, Paris, PUF.

Jakobson, Roman, 1962 [1939]. "Zur Struktur des Phonems », in Selected Writings 1, 's-Gravenhage, Mouton, 280-310.

- 1971 [1963]. Parts and Wholes in Language, in Selected Writings 2, 's-Gravenhage, Mouton, 280-284.

Jakobson, Roman \& Halle, Morris, 1956. « Phonology and Phonetics », in Fundamentals of Language, The Hague, Mouton, 1-51.

Lagache, Daniel, 1962. Structure en psychologie, in Bastide, 1962, 87-88.

Lepschy, Giulio, 1962. "Osservazioni sul termine "struttura" ", Annali della Scuola Normale Superiore di Pisa 31, 173-197 (repris in Id., Mutamenti di prospettiva nella linguistica, Bologna, Il Mulino, 1981, 37-71).

- 1976 [1966]. La linguistique structurale, Paris, Payot. 
Malinowski, Bronisław, 1923. "The Problem of Meaning in Primitive Languages », in Ogden, Charles K. et Richards, Ivor A., The Meaning of Meaning, London, Routledge \& Kegan Paul Ltd, 451-510.

Mathesius, Vilém, 1911. «Poznámky o tak zvané ellipse a anglických větách neslovesných » [Remarques sur ce qu'on appelle les ellipses et sur les énoncés anglais non verbaux], Sbornik Filologický 2, 215-234 (trad. it. « Note intorno alla cosiddetta ellissi e alle frasi senza verbo in inglese ", par Andrea Trovesi, introduction par S. Raynaud, Linguistica e Filologia 33, 2013, 127-162).

Mulligan, Kevin, 2004. «L'essence du langage, les maçons de Wittgenstein et les briques de Bühler», Friedrich, Janette et Samain, Didier (éd.), Karl Bühler. Science du langage et mémoire européenne, Dossiers d'HEL 2 (supplément électronique à la revue Histoire Épistémologie Langage), Paris, SHESL (http://htl.linguist.jussieu.fr/dosHEL.htm).

Nagel, Ernest, 1963 [1952]. «Wholes, Sums, and Organic Unities », in Lerner, Daniel (éd.), Parts and Wholes - The Hayden Colloquium on Scientific Method and Concept, New York, The Free Press of Glencoe, 135-155 (repris de Philosophical Studies III (2), 1952).

- 1979. The Structure of Science - Problems in the Logic of Scientific Explanation, Indianapolis, Hackett Publishing Company.

Petitot-Cocorda, Jean, 1985. Morphogenèse du sens, Paris, PUF.

Piaget, Jean, 1968. Le structuralisme, Paris, PUF.

Polanyi, Michael, 1958. Personal Knowledge - Towards a Post-Critical Philosophy, Chicago, Chicago University Press.

Pomian, Krzysztof, 1981. « Struttura », Enciclopedia, vol. 13, Torino, Einaudi, 723-764.

Pos, Hendrik J., 1939. « Perspectives du structuralisme », TCLP IX, 71-78.

Raynaud, Savina, 1990. Il Circolo Linguistico di Praga (1926-1939). Radici storiche e apporti teorici, Milano, Vita e Pensiero.

- 2012. Porre, comporre, disporre. Dai giudizi tetici agli enunciati tetici, ai temi e ai loro correlati, in Radimský, Jan (a cura di), Perspective fonctionnelle de la phrase - l'apport du Cercle de Prague, « Echo des études romanes » VIII (1) (volume thématique), 129141 (www.eer.cz/files/2012-1/2012-1-10-Raynaud.pdf).

Saussure, Ferdinand de, 1962 [1916]. Cours de linguistique générale, Paris, Payot.

- 1967-1968 ; 1974. Cours de linguistique générale. Édition critique établie par Rudolf Engler, Wiesbaden, Harrassowitz, 1967-1968 (fasc. 1-3), 1974 (fasc. 4).

Simons Peter, 1987. Parts - A Study in Ontology, Oxford, Oxford University Press.

Trubetzkoi, Nikolaj S., 1949 [1939]. Principes de phonologie, Paris Klincksieck.

Wegener, Philipp, 1991 [1885]. Untersuchungen über die Grundfragen des Sprachlebens, introduction par C. Knobloch, Amsterdam/Philadelphia.

Wittgenstein, Ludwig, 2005 [1953]. Recherches philosophiques, Paris, Gallimard. 